\title{
Electro-Mechanical Impedance Method for Crack Detection in Metallic Plates
}

\author{
Victor Giurgiutiu, Andrei Zagrai \\ Mechanical Engineering Department, University of South Carolina \\ Columbia, SC 29208, 803-777-8018, victorg@ sc.edu
}

\begin{abstract}
As a nondestructive evaluation technology, the EM impedance method allows us to identify the structural dynamics directly through in-situ active piezoelectric sensors. Previous work performed on 1-D steel beams structures shown through both theoretical analysis and experimental results that E/M impedance (or admittance) spectrum is a direct identifier of structural dynamics. The scope of presented work was to extend the positive results obtained for 1-D structure onto 2-D structures. Experiment analysis of 1-D and2-D structures has shown that E/M impedance (or admittance) spectrum accurately identifies the natural frequency spectrum of the specimens. Theoretical analysis was performed for particular boundary conditions to model the experimental set-up. Experiments were conducted on simple specimens in support of the theoretical investigation, and on thin-gauge aluminum plates to illustrate the method's potential. The number of specimens was sufficient to form a statistical data set. The aging aircraft panel was instrumented with piezoelectric active sensors and the spectrum of natural frequencies was measured at high frequency range. The changing of the spectrum due to presence of local small crack was noticed.
\end{abstract}

Keywords: impedance method, health monitoring, piezoelectric, circular plates, aging aircraft, cracks, delamination.

\section{INTRODUCTION}

Safe and efficient operation of structure is impossible without knowing its current structural health. Many nondestructive evaluation (NDE) technologies, each with its own area of expertise, could be used alone or together to archive this ultimate goal. Since knowing current, up-to-dated each reasonable time span, report on structural health is of great importance, the preference is often given for NDE technologies which are suitable for on-line structural health monitoring. Modal analysis and dynamic structural identification are widely used in many branches of science, engineering and industry for its relatively easy implementation, reliable results and capabilities of on-line monitoring. Structural frequencies, damping, and modes shapes identified through this process are subsequently used to predict dynamic response, avoid resonances, and even monitor structural change that are indicative of incipient failure (Harris, 1996).

Traditional modal analysis testing (Ewins, 1984; Maia et al, 1997; Heylen, 1997) relies on two essential components: (a) structural excitation; and (b) vibration pickups. The traditional structural excitation can be either harmonic sweep, or impulse. The former is more precise and can zoom in on resonant frequencies; the latter is more expedient and preferred for quick estimations. The vibration pickups can measure displacement, velocity, or acceleration. Current technologies cover miniaturized self-conditioning accelerometers Broch, (1984) and laser velocimeters (Polytec PI, Inc. 2000). The accelerometers allow installation of sensor arrays that accurately and efficiently measure the mode shapes, while the laser offers contactless measurements that are essential for low mass sensitive structures. The disadvantages of accelerometers are cost, unavoidable bulkiness, and possible interference with the structural dynamics through their added mass. Laser velocimeters, on the other hand, need to scan the structure to measure the mode shapes, and this significantly increases the duration of the experiments.

The advent of commercially available low-cost piezoceramics has opened new opportunities for structural identification. Through their intrinsic electro-mechanical (E/M) coupling, the piezoceramics can act as both sensors and actuators. Additionally, the frequency bandwidth of these sensors and actuators is orders of magnitude larger than that of conventional shakers and even impact hammers. Small piezoelectric (PZT) ceramic wafers can be permanently attached to structural surface. They could form sensor and actuator arrays that permit effective modal identification in a wide frequency band. Crawley and Luis (1987) proposed the use of piezoceramic wafers as elements of intelligent structures. Dimitiradis et al. (1991) and D'Cruz (1993) used piezoelectric wafers for structural excitation. Zhou et al. (1996) performed experiments in 
which a PZT wafer produced the excitation, while a laser velocimeter picked up the vibration response. Several investigators (Collins et al. (1992), Clark et al. (1993)) and others used piezo-polymer films for vibration sensing. Banks (1996) describes experiments in which the PZT wafer was used initially for excitation, and then for sensing the free decay response. Wang and Chen (2000) used a PZT wafer to excite the structure and an array of PVDF film sensors to pick up the forced vibration response to generate the frequencies and mode shapes through multi-point signal processing.

Liang et al. (1994) performed the coupled E/M analysis of adaptive systems driven by a surface-attached piezoelectric wafer. The aim of the analysis was to determine the actuator power consumption and system energy transfer. A 1-degree of freedom (1-DOF) analysis was performed, and the electrical admittance as measure at the terminals of the PZT wafer attached to the structure was derived in the form:

$$
Y(\omega)=i \omega C\left(1-\kappa_{31}^{2} \frac{Z_{s t r}(\omega)}{Z_{s t r}(\omega)+Z_{A}(\omega)}\right)
$$

where $C$ is the electrical capacitance of the PZT active sensor, $Z_{s t r}(\omega)$ is the 1-DOF structural impedance as seen by the sensor, and $Z_{A}(\omega)$ is the quasi-static impedance of the sensor. However, no modeling of the structural substrate was included, and no prediction of $Z_{s t r}(\omega)$ for a multi-DOF structure was presented. This work was continued and extended by Sun $e t$ al. (1994) who used the half-power bandwidth method to accurately determine the natural frequency values. Mode shape extraction methodology, using multiple sensors self and across admittances were explored. Experiments were performed on aluminum beams at frequencies up to $7 \mathrm{kHz}$. These two papers were the first to conceptualize that the E/M admittance as seen at the sensor terminals reflects the coupled-system dynamics, and that an embedded PZT wafer could be used as structural-identification sensor. However, no theoretical modeling of the E/M impedance/admittance response for comparison with experimental data was attempted.

Subsequently, several authors reported the use of the E/M impedance method for structural health monitoring, whereby the admittance or impedance frequency spectra of pristine and damaged structures were compared (Chaudhry et al $(1994,1995)$, Ayres et al (1996), Giurgiutiu (1998)). The method has been shown to be especially effective at ultrasonic frequencies, which properly capture the changes in local dynamics due to incipient structural damage. (Such changes are too small to affect the global dynamics and hence cannot be readily detected by conventional low-frequency vibration methods). The method is direct and easy to implement, the only required equipment being an electrical impedance analyzer.

In this paper, the scope of theoretical analysis and experimental results for 1-D steel beams structures are presented. The positive results obtained for 1-D structure are extended onto 2-D aluminum plates structures. Circular thin aluminum plates are considered. Theoretical analysis was performed for particular boundary conditions to model the experimental set-up. The spectrum of natural frequencies was measured at high frequency range for pristine circular plates and aging aircraft panel in which changing of the spectrum due to presence of local small crack was noticed.

\section{ONE DIMENSIONAL STRUCTURES}

To better understand the relationship between structural resonances and the frequencies displayed by the E/M admittance/impedance spectrum of the PZT active sensor attached to the structure we performed experiments on simple beam and plate specimens. Structural modeling, performed in parallel, was used to predict the structural resonance frequencies and mode shapes. The theoretical and experimental results were compared, and validation of the theoretical prediction was achieved.

One-dimensional beam structures are easy to model, and the prediction of their natural frequencies is fairly well understood (Inman, 1996). The effect of structural and sensor damping can be easily introduced (Giurgiutiu and Zagrai, 2001a) by the use of complex notations. In this case, the following expressions could be used to model of E/M admittance/impedance response seen by a sensor attached to structure.

$$
\bar{Y}=\mathrm{i} \omega \cdot \bar{C}\left[1-\bar{\kappa}_{31}^{2}\left(1-\frac{1}{\bar{\varphi} \cot \bar{\varphi}+\bar{r}}\right)\right] \bar{Z}=\frac{1}{\mathrm{i} \omega \cdot \bar{C}}\left[1-\bar{\kappa}_{31}^{2}\left(1-\frac{1}{\bar{\varphi} \cot \bar{\varphi}+\bar{r}}\right)\right]^{-1}
$$

where $\bar{r}$ is the frequency-dependent complex stiffness ratio that reflects the structural point-wise dynamics and the sensor dissipation mechanisms. 


\subsection{Modeling of a PZT Active Sensor Installed on a Structural Substrate}

The dynamics of the structural substrate and its interaction with the sensor dynamics is paramount of following analysis. To couple the sensor and the structure, we are going to use the dynamic structural stiffness, $k_{s t r}(\omega)$, which could be calculated using the general theory of structural vibrations (Timoshenko, 1955; Meirovitch, 1986; Inman, 1996; and Kelly, 2000). However, the PZT excitation departs from the typical textbook formulation since it acts a pair of self-equilibrating axial forces and bending moments that are separated by a small finite distance, $l_{P Z T}$. This feature gives gusto to our analysis.

\subsubsection{Definition of the Excitation Forces and Moments}

The excitation forces and moments acting upon the beam structure are derived from the PZT force, $F_{P Z T}=\hat{F}_{P Z T} e^{i \omega t}$, using the beam cross-section geometry (Giurgiuiu and Zagrai, 2001a):

$$
M_{a}=F_{P Z T} \frac{h}{2}, \quad N_{a}=F_{P Z T}
$$

The space-wise distribution of excitation bending moment and axial force are expressed using the Heaviside function, $H(x-$ $\left.x_{a}\right)$, defined as $H\left(x-x_{a}\right)=0$ for $x<x_{a}$, and $H\left(x-x_{a}\right)=1$ for $x_{a} \leq x$ :

$$
\begin{gathered}
N_{e}(x, t)=N_{a}\left[-H\left(x-x_{a}\right)+H\left(x-x_{a}-l_{a}\right)\right] \cdot e^{i \omega t} \\
M_{e}(x, t)=-M_{a}\left[-H\left(x-x_{a}\right)+H\left(x-x_{a}-l_{a}\right)\right] \cdot e^{i \omega t}
\end{gathered}
$$

Equations (4) and (5) correspond to axial and flexural vibrations, respectively. Axial vibrations modes are usually of much larger frequency than flexural vibration modes, and were neglected by previous researchers (Liang et al., 1994). However, their vibration frequencies are commensurable with those of the PZT active sensors. Other researchers have only considered axial modes and neglected the flexural vibrations (Park, Cudney and Inman, 2000).

\subsubsection{Calculation of Frequency Response Function and Dynamic Structural Stiffness}

To obtain the dynamic structural stiffness, $k_{s t r}$, presented by the structure to the PZT, we first calculate the elongation between the two points, A and B, connected to the PZT ends. Simple kinematics gives the horizontal displacement of a generic point $P$ place on the surface of the beam:

$$
u_{P}(t)=u(x)-\frac{h}{2} w^{\prime}(x),
$$

where $u$ and $w$ are the axial and bending displacements of the neutral axis. Letting $\mathrm{P}$ be $\mathrm{A}$ and $\mathrm{B}$, and taking the difference, yields:

$$
u_{P Z T}(t)=u_{B}(t)-u_{A}(t)=u\left(x_{a}, t\right)-u\left(x_{a}+l_{a}, t\right)-\frac{h}{2}\left[w^{\prime}\left(x_{a}, t\right)-w^{\prime}\left(x_{a}+l_{a}, t\right)\right]
$$

Using classical analysis of axial and flexural vibrations (Giurgiutiu and Zagrai, 2001b) Equation (7) becomes

$$
\hat{u}_{P Z T}=\frac{\hat{F}_{P Z T}}{\rho A}\left\{\sum_{n_{u}} \frac{\left[U_{n_{u}}\left(x_{a}+l_{a}\right)-U_{n_{u}}\left(x_{a}\right)\right]^{2}}{\omega_{n_{u}}^{2}-\omega^{2}}+\left(\frac{h}{2}\right)^{2} \sum_{n_{w}} \frac{\left[W_{n_{w}}^{\prime}\left(x_{a}+l_{a}\right)-W_{n_{w}}^{\prime}\left(x_{a}\right)\right]^{2}}{\omega_{n_{w}}^{2}-\omega^{2}}\right\}
$$

where differentiation between axial and flexural vibrations frequencies and mode shapes was achieved by the use of $n_{u}, \omega_{n_{u}}$, $U_{n_{u}}(x)$ and $n_{w}, \omega_{n_{w}}, W_{n_{w}}(x)$, respectively. Dividing Equation (8) by $\hat{F}_{P Z T}$ yields the structural frequency response function (FRF) to the Single Input Single Output (SISO) excitation applied by the PZT active sensor. This situation is similar to conventional modal testing (Harris, 1996, Section 21) with the proviso that the PZT wafers are unobtrusive and permanently attached to the structure. The dynamic structural stiffness is the inverse of frequency response function, i.e., 


$$
k_{s t r}(\omega)=\frac{\hat{F}_{P Z T}}{\hat{u}_{P Z T}}=\rho A\left\{\sum_{n_{u}} \frac{\left[U_{n_{u}}\left(x_{a}+l_{a}\right)-U_{n_{u}}\left(x_{a}\right)\right]^{2}}{\omega_{n_{u}}^{2}+2 \mathrm{i} \zeta_{n_{u}}-\omega^{2}}+\left(\frac{h}{2}\right)^{2} \sum_{n_{w}} \frac{\left[W_{n_{w}}^{\prime}\left(x_{a}+l_{a}\right)-W_{n_{w}}^{\prime}\left(x_{a}\right)\right]^{2}}{\omega_{n_{w}}^{2}+2 \mathrm{i} \zeta_{n_{w}}-\omega^{2}}\right\}^{-1}
$$

For free-free beams (Inman, 1996) axial and flexural components are:

$$
\begin{aligned}
& U_{n_{u}}(x)=A_{n_{u}} \cos \left(\gamma_{n_{u}} x\right), A_{n_{u}}=\sqrt{2 / l}, \gamma_{n_{u}}=\frac{n_{u} \pi}{l}, \omega_{n_{u}}=\gamma_{n_{u}} c, c=\sqrt{\frac{E}{\rho}}, n_{u}=1,2, \ldots \\
& W_{n_{w}}(x)=A_{n_{w}}\left[\cosh \gamma_{n_{W}} x+\cos \gamma_{n_{w}} x-\sigma_{n_{w}}\left(\sinh \gamma_{n_{w}} x+\sin \gamma_{n_{w}} x\right)\right] \omega_{n_{w}}=\gamma_{n_{w}}^{2} a, a=\sqrt{\frac{E I}{\rho A}}, A_{n_{w}}=1 / \sqrt{\int_{0}^{l} W_{n_{w}}^{2}(x) d x}
\end{aligned}
$$

Numerical values of $l \cdot \gamma_{n_{w}}$ and $\sigma_{n_{w}}$ for $n_{w} \leq 5$ can be found in Blevins (1979), page 108; for $5<n_{w}, \gamma_{n_{w}}=\frac{1}{l} \frac{(2 n+1) \pi}{2}$ and $\sigma_{n_{w}}=1$. The derivations (2), (9), (10), (11) open the way for comparison between theoretical and experimental results.

\subsection{Experimental Results for One-dimensional Structures}

To validate theoretical assumptions a number of small steel beams various thickness and width values were fabricated for experimental investigation. All beams were 100mm long, but their width varied from $19.6 \mathrm{~mm}$ (wide beams) to $8 \mathrm{~mm}$ (narrow beams). The nominal thickness of the specimen was $5.2 \mathrm{~mm}$; by gluing two specimens back-to-back, we were also able to create double thickness specimens. Thus, four beam types were used (Figure 1a): narrow-thin, narrow-thick, wide-thin, and wide-thick. The comparison of wide and narrow beams was aimed at identifying the width effects in the frequencies spectrum, while the change from double to simple thickness was aimed at simulating the effect of corrosion (for traditional structures) and disbonding/delamination on adhesively bonded and composite structures. All specimens were instrumented with 7-mm square PZT active sensors placed at $40 \mathrm{~mm}$ from one end.
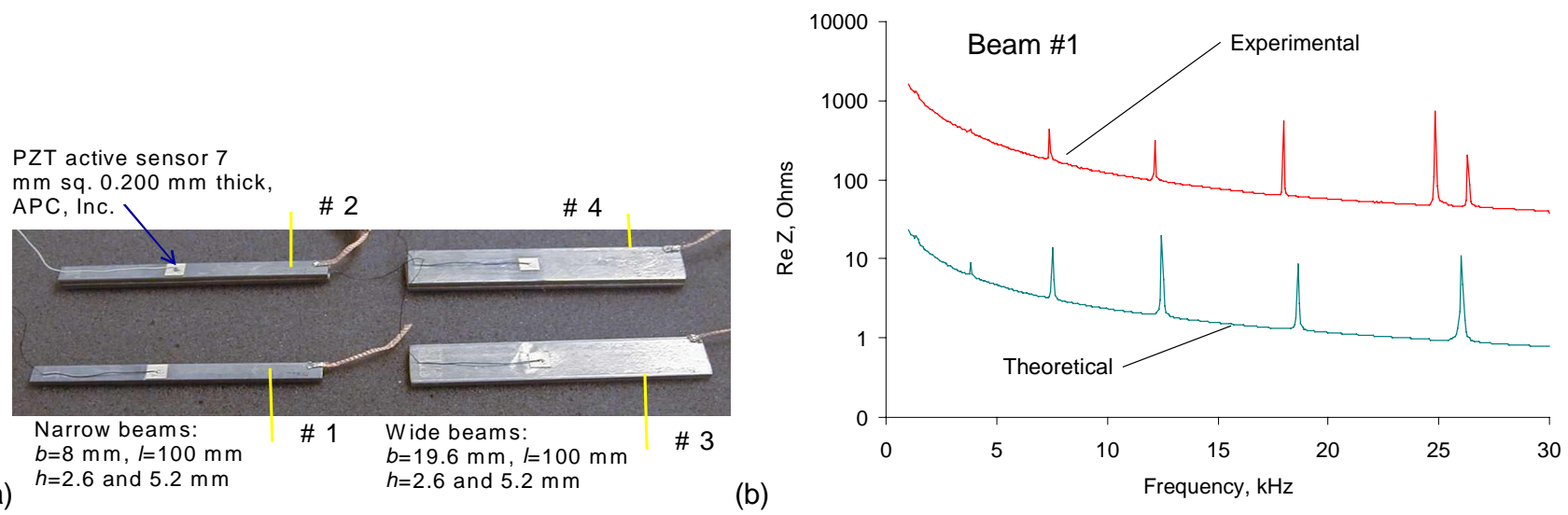

Figure 1 Test specimens (a) and experimental vs. theoretical results (b) for one dimension structure simulated with steel beams.

During the experiments, recording of the E/M impedance real part spectrum with the HP 4194A Impedance Analyzer was performed in the $1-1000 \mathrm{kHz}$. When necessary, frequency zoom was employed. To approximate the free-free boundary conditions, the beams were supported on common packing foam. The beam natural frequencies were identified from the E/M impedance spectrum. The theoretical analysis (Giurgiutiu and Rogers, 2000b) indicates that these frequencies should be identical with the basic beam resonances, as predicted by classical vibration analysis (Inman, 1996). This was numerically confirmed using a MathCAD-coded simulation program. Free-free boundary conditions, and the associated mode shapes and frequency expressions (Inman 1996) were used during the simulations. When the beam thickness was doubled, the frequencies also doubled.

An example of the actual impedance spectra is given in Figure 1b. The calculated and measured results are shown superposed (to fit into same graph, selective scaling was applied). Since these graphs refer to single-thickness beams, the first 5 flexural resonances are contained in the $1-20 \mathrm{kHz}$ range. For the first 4 modes, the predicted and measured frequency values almost superpose. For the $5^{\text {th }}$ mode, there is a slight difference. This proves again that the predicted and measured results are in close agreement, well within the tolerance normally expected from experimental modal analysis. 


\section{TWO DIMENSIONAL STRUCTURES}

Metallic plates are common members of complex civil, marine and aircraft structures. Thus, knowing its dynamic state is important for maintaining structural integrity, safety and health. In this section we overview the classical vibration theory used in structural identification and present solutions for various boundary conditions at the outer radius of thin circular plates.

The theoretical foundation for transverse vibrations of isotropic circular plates was first published by Airey at 1911 and extended by Colwell et al, (1936). Since then, many authors in various braches of science and engineering formulate and solve vibration problems for specific cases. An outstanding overview of a subject was presented by Leissa, (1969). The theoretical background and numerical results were given for large variety of boundary conditions and plate shapes. However, for complete developments reader should address other references. (Wah, (1962); Kunukkasseril and Swamidas, (1974); Soedel, (1993); Rao (1999)). The generic treatment of transversely vibrating circular plates usually consider following equation:

$$
D \cdot \nabla^{4} w+K \cdot w+\rho h \frac{\partial^{2} w}{\partial t^{2}}=q(r, t)
$$

where $D=\frac{E h^{3}}{12 \cdot\left(1-v^{2}\right)}$ is the flexural rigidity, $K$ is the stiffness of the elastic foundation or support, $q(r, t)$ is an excitation function and $\rho, h$ are density and thickness of a plate. Operator $\nabla^{4}=\nabla^{2} \nabla^{2}$ where $\nabla^{2}$ - Laplacian operator polar coordinates is

$$
\nabla^{2}=\frac{\partial^{2}}{\partial r^{2}}+\frac{1}{r} \frac{\partial}{\partial r}+\frac{1}{r} \frac{\partial^{2}}{\partial \theta^{2}}
$$

It is well known that solution for free $(q(r, t)=0)$ and forced vibration of circular plates is introduced in terms of Bessel functions of different orders. For a circular plate closed in $\theta$ direction the order of Bessel functions should be integer (not rational) and for $n=0,1,2,3 \ldots$ space-wise solution of Equation (12) $(K=0)$ is:

$$
W(r, \theta)={ }_{\sin (n \cdot \theta)}^{\cos (n \cdot \theta)} \sum_{n=0}^{\infty} C_{n} J_{n}(\lambda r)+D_{n} I_{n}(\lambda r)+E_{n} Y_{n}(\lambda r)+F_{n} K_{n}(\lambda r)
$$

where $J_{n}, Y_{n}$ are Bessel functions of first and second kinds and $I_{n}, K_{n}$ are modified Bessel function of first and second kinds. For a plate with no central hole $E=F=0$. Other constants are determined by imposition of particular set of boundary conditions. We consider example of clamped and free-free boundary conditions at the outer radius $a$ of a plate.

\subsection{Clamped Boundary Conditions}

For plates clamped all around following relationship holds

$$
W(a)=0 \text { and } \frac{\partial W(a)}{\partial r}=0
$$

Substituting these boundary conditions into simplified Equation (14) leads to frequency equation

$$
J_{n}(\lambda a) \frac{d I_{n}(\lambda a)}{d r}-I_{n}(\lambda a) \frac{d J_{n}(\lambda a)}{d r}=0
$$

which gives values for $\lambda a$ tabulated in various sources (Leissa, (1969), Soedel (1993)). Once these roots are known for desired number of circles $m$ and diameters $n$ i.e. particular mode-shape, calculation of natural frequencies is straightforward.

$$
\omega_{m n}=\frac{(\lambda a)_{m n}^{2}}{a^{2}} \sqrt{\frac{D}{\rho h}}
$$

Further development of Equation (16) yields expression for vibration mode-shapes.

$$
W(r, \theta)=\frac{\cos (n \cdot \theta)}{\sin (n \cdot \theta)} \sum_{n=0}^{\infty} A_{m n} \cdot\left[J_{n}\left(\frac{\lambda a_{m n}}{a} r\right)-\frac{J_{n}\left(\lambda a_{m n}\right)}{I_{n}\left(\lambda a_{m n}\right)} I_{n}\left(\frac{\lambda a_{m n}}{a} r\right)\right]
$$


Equation (18) is plotted in 3-D on a Figure 2 for a case of zero nodal diameters $(n=0)$
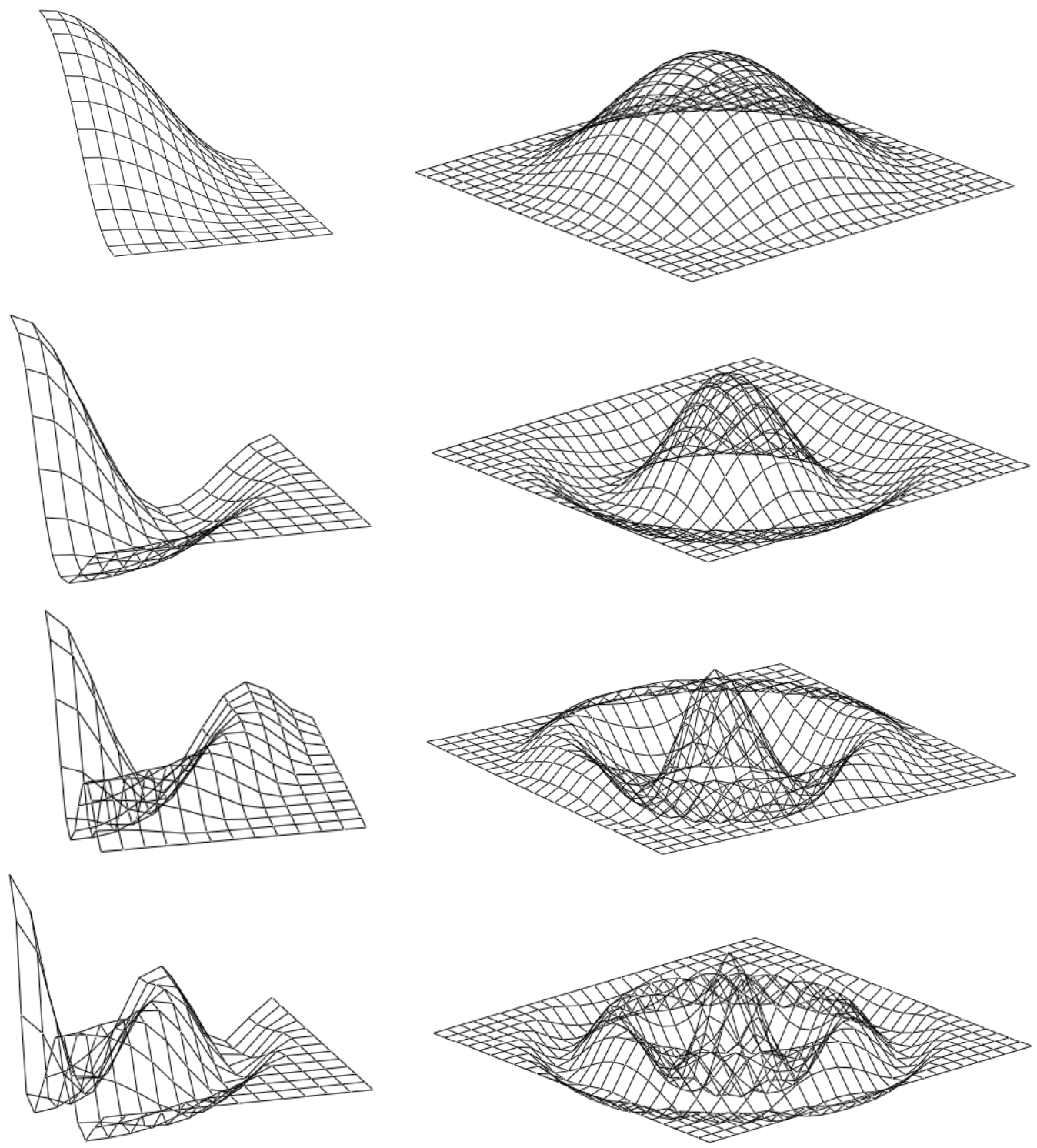

Figure 2 First four mode-shapes (zero nodal diameters, $n=0$ ) of circular plate clamped at the outer radius.

\subsection{Free-Free Boundary Condition}

Free-free boundary condition is not easy to model since no analytical solution exist. Boundary conditions are governed by following expressions:

$$
M_{r}(a)=0 \quad \text { and } \quad V_{r}(a)=0
$$

Using this set of boundary condition, frequency equation could be obtained .

$$
\begin{aligned}
C_{m n}= & \frac{\lambda a_{m n}^{2} \cdot J_{n}\left(\lambda a_{m n}\right)+(1-v) \cdot\left[\lambda a_{m n} \cdot J_{n}^{\prime}\left(\lambda a_{m n}\right)-n^{2} \cdot J_{n}\left(\lambda a_{m n}\right)\right]}{\lambda a_{m n}^{2} \cdot I_{n}\left(\lambda a_{m n}\right)-(1-v) \cdot\left[\lambda a_{m n} \cdot I_{n}^{\prime}\left(\lambda a_{m n}\right)-n^{2} \cdot I_{n}\left(\lambda a_{m n}\right)\right]}= \\
= & \frac{\lambda a_{m n}^{3} \cdot J_{n}^{\prime}\left(\lambda a_{m n}\right)+n^{2} \cdot(1-v) \cdot\left[\lambda a_{m n} \cdot J_{n}^{\prime}\left(\lambda a_{m n}\right)-J_{n}\left(\lambda a_{m n}\right)\right]}{\lambda a_{m n}^{3} \cdot I_{n}^{\prime}\left(\lambda a_{m n}\right)-n^{2} \cdot(1-v) \cdot\left[\lambda a_{m n} \cdot I_{n}^{\prime}\left(\lambda a_{m n}\right)-I_{n}\left(\lambda a_{m n}\right)\right]}
\end{aligned}
$$


Frequency parameter $\lambda a_{m n}$ and mode-shape parameter $C_{m n}$ are obtained numerically by solving the eigenvlue problem described above are tabulated by Itao and Crandall, (1979) for 701 modes. In addition, the same reference features an amplitude parameter $A_{m n}$ obtained through normalization process. Using Equation (17) natural frequencies could be determined according to the values of frequency parameter presented by (Itao and Crandall, (1979)). The mode-shape equation yields:

$$
W(r, \theta)=\left\{\begin{array}{c}
A_{m n} \cdot\left[J_{n}\left(\frac{\lambda a_{m n}}{a} r\right)+C_{m n} \cdot I_{n}\left(\frac{\lambda a_{m n}}{a} r\right)\right] \cdot \sin (n \cdot \theta), n<0 \\
A_{m 0} \cdot\left[J_{0}\left(\frac{\lambda a_{m 0}}{a} r\right)+C_{m 0} \cdot I_{0}\left(\frac{\lambda a_{m 0}}{a} r\right)\right], n=0 \\
A_{m n} \cdot\left[J_{n}\left(\frac{\lambda a_{m n}}{a} r\right)+C_{m n} \cdot I_{n}\left(\frac{\lambda a_{m n}}{a} r\right)\right] \cdot \cos (n \cdot \theta), n>0
\end{array}\right.
$$

Example of mode-shapes for free-free boundary condition and arbitrary radius is given on Figure 3. The mode-shapes were calculated with Equation (21) for a case of zero nodal diameters.

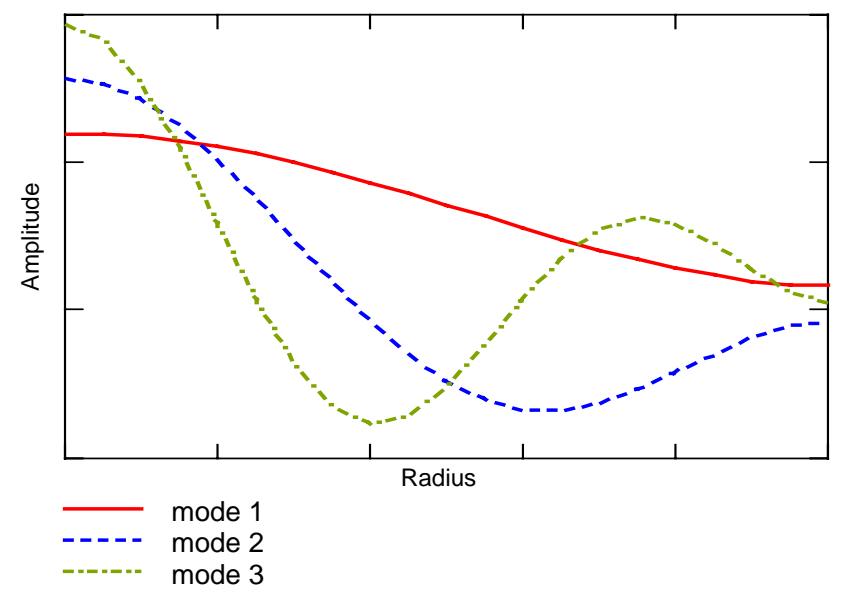

Figure 3 First three mode-shapes for circular plate with free-free boundary condition.

\section{EXPERIMENTS WITH PRISTINE PLATES}

Since plates are common members of aircraft structures a series of experiments on thin-gage aluminum plates were conducted to understand how application of E/M impedance method could be transitioned from 1-D beam structures into 2-D plate structures. Twenty-five plate specimens (100-mm square and circular, 1-mm thick) were constructed from aircraft-grade aluminum stock. Each plate was instrumented with one 7-mm square and circular PZT active sensor placed at its center (Figure 4). Data was taken on 5 of these identical specimens using the HP 4194A Impedance Analyzer.
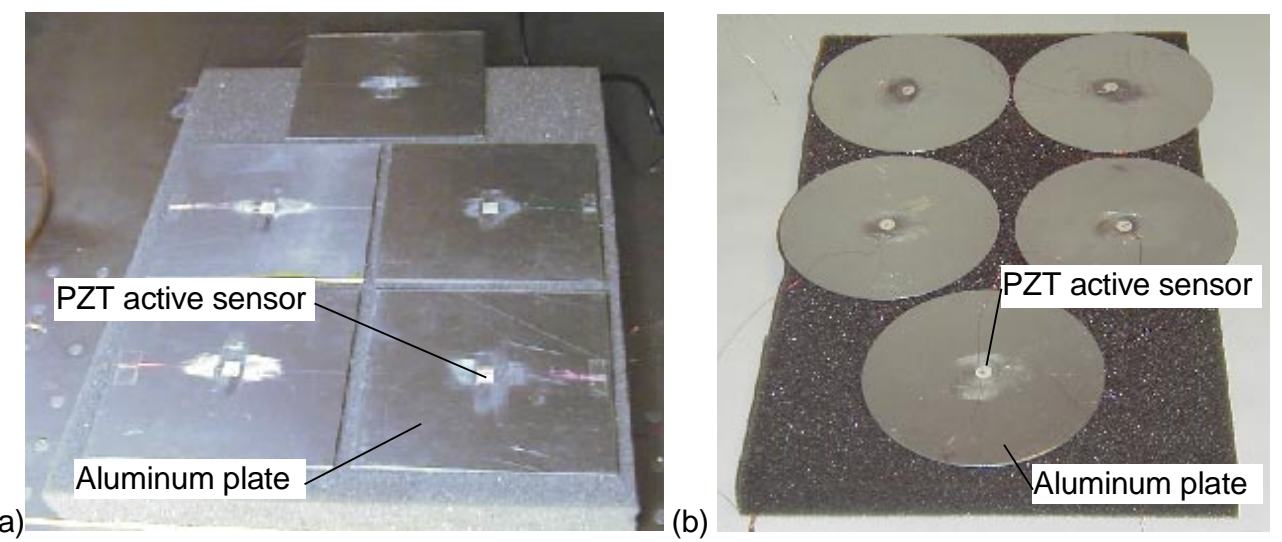

Figure 4 Thin-gage aluminum plate specimens with centrally located piezoelectric sensors: (a) 100-mm square plates, thickness $1 \mathrm{~mm}$; (b) 100-mm circular plates, thickness $-0.8 \mathrm{~mm}$. 
Table 1 Experimental results for square aluminum plates

\begin{tabular}{|c|c|c|c|c|c|c|c|c|}
\hline $\begin{array}{c}\text { Mode } \\
\text { number }\end{array}$ & $\begin{array}{c}\text { Plate 6 } \\
\text { frequency } \\
\mathbf{k H z}\end{array}$ & $\begin{array}{c}\text { Plate 7 } \\
\text { frequency } \\
\mathbf{k H z}\end{array}$ & $\begin{array}{c}\text { Plate 8 } \\
\text { frequency } \\
\mathbf{k H z}\end{array}$ & $\begin{array}{c}\text { Plate 9 } \\
\text { frequency } \\
\mathbf{k H z}\end{array}$ & $\begin{array}{c}\text { Plate 10 } \\
\text { frequency } \\
\mathbf{k H z}\end{array}$ & $\begin{array}{c}\text { Mean } \\
\text { frequency } \\
\mathbf{k H z}\end{array}$ & $\begin{array}{c}\text { Standard } \\
\text { deviation } \\
\mathbf{k H z}\end{array}$ & $\begin{array}{c}\text { Standard } \\
\mathbf{d e n} \\
\mathbf{\text { deviation }} \\
\mathbf{\%}\end{array}$ \\
\hline 3 & 5.7500 & 5.7125 & 5.7875 & 5.7125 & 5.7875 & 5.7500 & 0.0375 & $0.6522 \%$ \\
\hline 4 & 6.2375 & 6.2375 & 6.2750 & 6.2750 & 6.3125 & 6.2675 & 0.0314 & $0.5006 \%$ \\
\hline 5 & 7.0250 & 7.0250 & 7.0650 & 6.9875 & 7.1375 & 7.0480 & 0.0570 & $0.8094 \%$ \\
\hline 6 & 9.5750 & 9.5375 & 9.6500 & 9.6125 & 9.6125 & 9.5975 & 0.0428 & $0.4455 \%$ \\
\hline 7 & 11.5620 & 11.5250 & 11.6375 & 11.6000 & 11.6375 & 11.5924 & 0.0490 & $0.4224 \%$ \\
\hline 8 & 12.3500 & 12.2750 & 12.4250 & 12.3875 & 12.4625 & 12.3800 & 0.0721 & $0.5827 \%$ \\
\hline 9 & 13.4375 & 13.4000 & 13.5125 & 13.5125 & 13.5125 & 13.4750 & 0.0530 & $0.3936 \%$ \\
\hline 10 & 14.8625 & 14.8250 & 14.9375 & 14.9750 & 15.0125 & 14.9225 & 0.0778 & $0.5211 \%$ \\
\hline 11 & 18.8375 & 18.7250 & 18.9875 & 18.9500 & 18.9875 & 18.8975 & 0.1144 & $0.6052 \%$ \\
\hline 12 & 19.2875 & 19.3625 & 19.4375 & 19.3250 & 19.6250 & 19.4075 & 0.1336 & $0.6886 \%$ \\
\hline
\end{tabular}

During the experiments, the specimens were supported on commercially available packing foam to simulate free-free conditions. Plate resonance frequencies were identified from the E/M impedance real part spectra. Figure 5a presents a typical case for the identification of the $6^{\text {th }}$ plate frequency. Superposed in Figure $5 \mathrm{a}$ are the spectrum peaks from 5 identical plates. It can be appreciated that they fall in a narrow frequency band, with mean and standard deviation values of $9.952 \mathrm{kHz}$, and $\pm 0.577 \%$, respectively. The corresponding bell-shaped statistics is given in Figure 5b. Similar results were also obtained for the higher frequencies. The resonance-frequency data was statistically processed, and the mean and standard deviation of each resonance frequency were computed (Table 1). Analysis of the experimental data presented in Table 1 indicates that frequency identification of thin-gage metallic plates using the E/M impedance method and PZT active sensors can be achieved consistently and with a good repeatability.

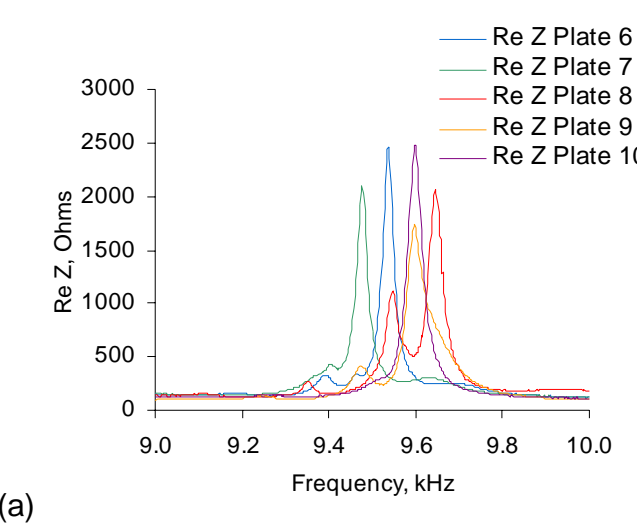

(b)

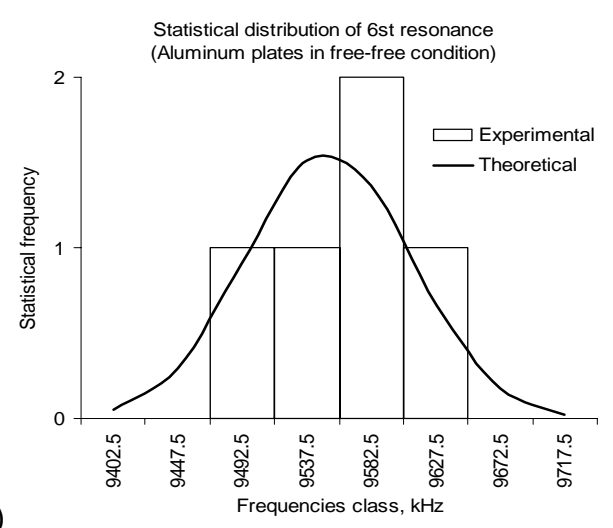

Figure 5 (a) E/M impedance spectrum in the vicinity of the $6^{\text {th }}$ plate resonance; (b) bell-shape statistics for 5 plates.

In order to validate the assumptions used for modeling of 1-D structures, the estimation of natural frequencies of plates and experimental confirmation is needed. Using Equation (17) and numerical values for frequency parameter (Itao and Crandall, (1979)) natural frequencies were calculated for free-free case of boundary conditions of circular plates. The obtained numerical results were entered in Table 2. For experimental validation, we consider five specimens. Each specimen consisted of $0.8 \mathrm{~mm}$ thick aluminum circular plate with diameter of $100 \mathrm{~mm}$. The piezoelectric strain sensor of circular shape was attached in the middle of each specimen. Since strains sensors are sensitive to changing in curvature, the vibration modes, which have maximum curvature at the middle of a plate, should be most successfully determined. In this case, the experimental set up gives us results for zero nodal diameters, $n=0$, condition. This assumption perfectly match with experimental and calculated results presented in Table 2. From another hand, any misalignment of a sensor relative to the center of a plate will give additional frequencies in the spectrum due to presence of other harmonics described with higher order Bessel functions. These harmonics, however, have little effect on frequency spectrum when the sensor is properly aligned. In this case, the solution should be presented in terms of Bessel functions of zero order, which significantly contributes to the spectrum, as it is shown on Figure 6. 


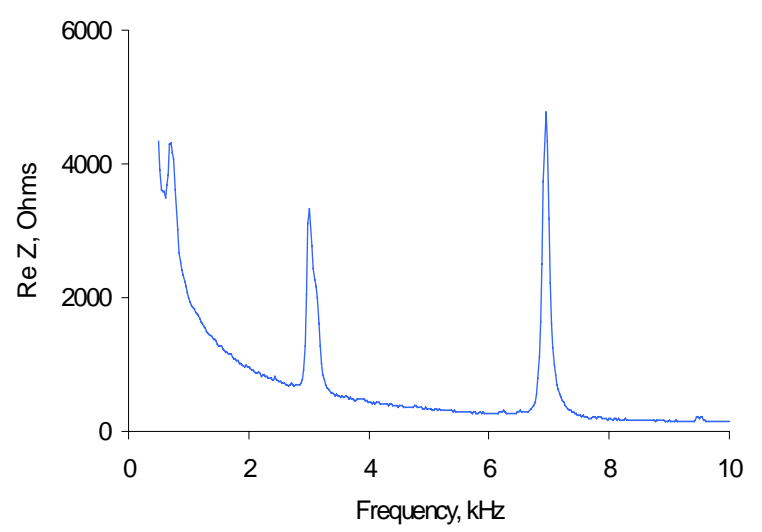

Table 2 Theoretical and experimental results for first 3 natural frequencies of circular plate \#2.

Figure 6 Experimental spectra of $\mathrm{E} / \mathrm{M}$ impedance of the circular plate \#2

\section{EXPERIMENTS WITH CRACKED PLATES}

The experiments with cracked plates are currently under development. We would like to present details on design of the specimens, which will be used further investigation. The first step of experiments includes fabrication of aluminum plates and simulation of cracks using an EDM process. The purpose of this process is to simulate cracks as often happen in the aircraft panels under loads. The strategy is to first affix the sensors on the plates and determine the E/M impedance spectra under pristine conditions (Figure $4 \mathrm{~b}$ and Figure 6). The spectral features will be compared for consistency since the plates and sensors are virtually identical. After this initial evaluation, the plates will be subjected to EDM processing. Two sets of specimens with EDM processed slits will be investigated.

\subsection{Specimen set \#1}

Specimen set \#1 consists of 5 groups of 5 plates (Figure 7). The first group will have simulated crack very close to the PZT active sensor. The second group will have the slits further away the third group even further away, while the fourth group will have the crack right at the edge of the plate. It was assumed that the fourth group should have crack with practically so far away from the PZT sensor's that they is out of sensor's area of sensitivity. The fifth group have no simulated cracks at all, but will be, nevertheless, immersed in the EDM fluid for a period of time equal to that experienced by the other groups. In this way, the effect, if any, of the EDM fluid on the sensors and their adhesive was evaluated. After the EDM processing, the specimens were subjected to the E/M impedance measurements, and the resulting spectra was compared with the baseline spectra measured on pristine specimens.

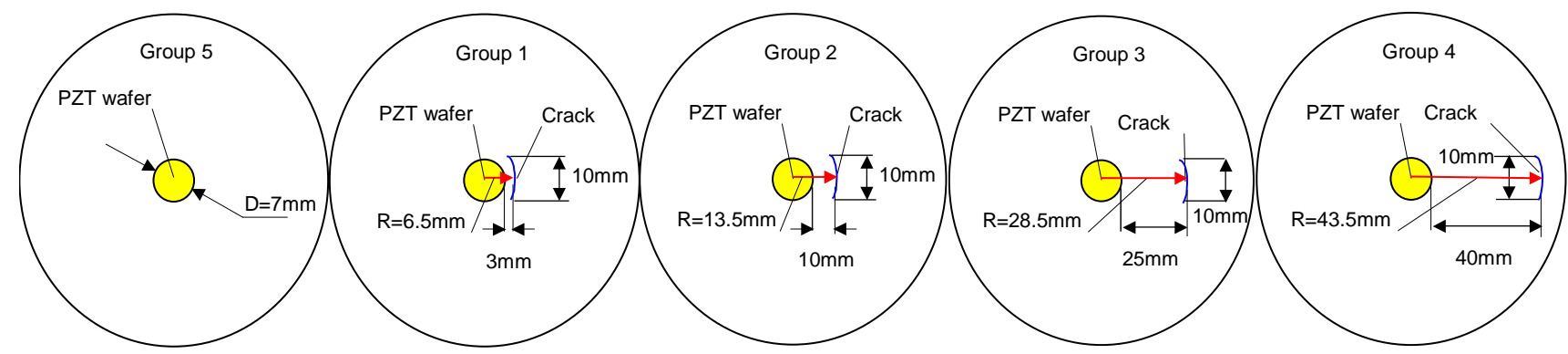

Figure 7 Specimen set \#1 details on slits dimensions and location.

\subsection{Specimen set \#2}

Specimen set \#2 consist of 5 plates. The first plate will have the simulated crack grooving from the edge of a plate towards sensor and come very close it. The second plate will have the slits further towards sensor, the third group even further towards sensor, while the fourth group will have the slit vicinity right next to the edge of the PZT active sensor. The fifth group will have no simulated cracks at all, but will be immersed in the EDM fluid for a period of time equal to that experienced by the other groups. Details on slit are given on Figure 8. 


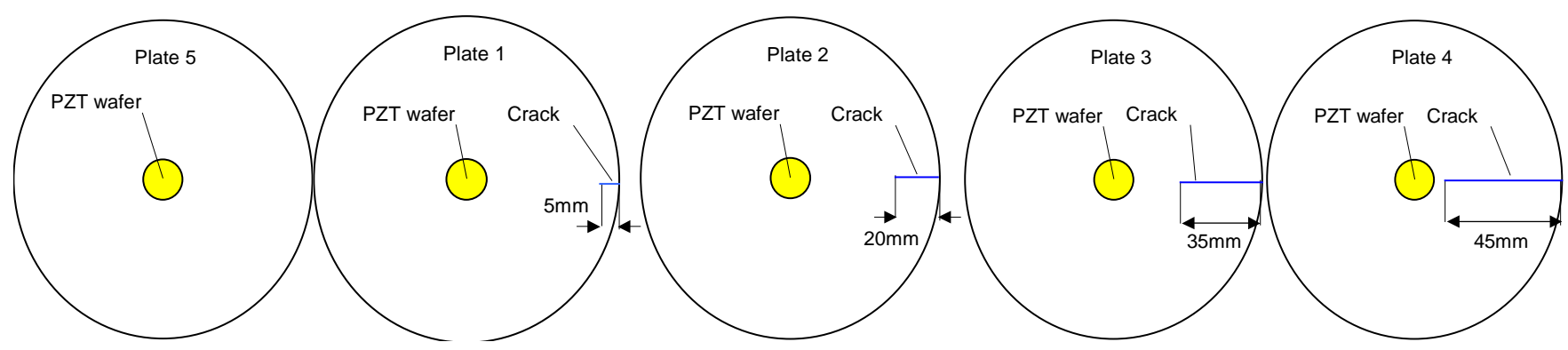

Figure 8 Specimen set \#2 details on slits dimensions and location.

\section{EXPERIMENTS WITH AGING AIRCRAFT PANEL}

Realistic aerospace panel specimens containing simulated crack and corrosion damage representative of aging-aircraft structures, designed and constructed at Sandia National Labs (Giurgiutiu et al., 2000a), were instrumented with PZT active sensors and subjected to E/M impedance evaluation. The results obtained during the present investigation were compared with previous results (Giurgiutiu et al., 2000a) obtained during investigations in which standardized sensor fabrication and installation procedures were not yet available. The sensors were applied to the simulated aircraft panels to detect the change of E/M impedance spectrum induced by the proximity of a simulated crack. Figure 9a shows sensors installation: the sensors are placed along a line, perpendicular to a $10-\mathrm{mm}$ crack originating at a rivet hole. The sensors are $7-\mathrm{mm}$ square and are spaced at 7-mm pitch.
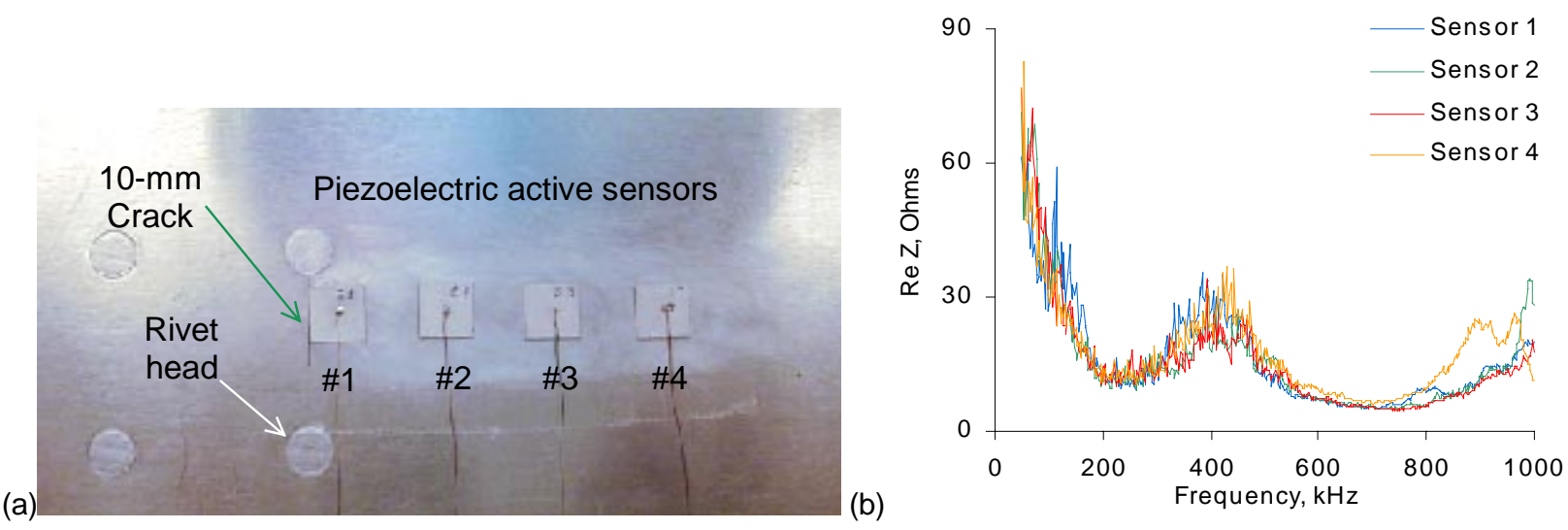

Figure 9 (a) Piezoelectric sensors installed on the aircraft panel with aging damage simulated by a 10-mm crack originating from a rivet; (b) Real part of impedance for sensors bonded on aging aircraft structure (zoom into the 50-1000 kHz range).

E/M impedance readings were taken of each sensor in the $50-1000 \mathrm{kHz}$ range. Figure $9 \mathrm{~b}$ shows the frequency spectrum of the E/M impedance real part. The spectrum reflects clearly defined resonances that are indicative of the coupled dynamics between the PZT sensors and the frequency-dependent pointwise structural stiffness as seen at each sensor location. Examination of Figure 9b indicates that, out of the four E/M impedance spectra, that of sensor 1 (closest to the crack) presents an additional frequency peak at $114 \mathrm{kHz}$ that is not present in the other sensors. It also shows a downward shift of the $400 \mathrm{kHz}$ main peak. These features are indicative of a correlation between the particularities of sensor 1 spectrum and the fact that sensor 1 is placed closest to the crack. However, at this stage of the investigation, these correlations are not self evident since experimental set up for plates with simulated cracks is under development. nor are they supported by of the structure under consideration. For these reasons, we conclude that, until experimental evidence will be obtained, further emphasis will be put on theoretical analysis and predictive modeling to fully understand the correlation between the spectral features of the E/M impedance response and the presence of structural damage in the sensor vicinity.

\section{CONCLUSION}

The application of E/M impedance method for various types of structures was considered in this paper. The quasi-static approach previously presented by Liang et al. (1994) was extended and derived mathematical expressions accounted for the 
dynamic response of both the sensor and the structure. The structural dynamics was incorporated through the pointwise dynamic stiffness presented by the structure to the sensor. The analytical model, developed for 1-D structures, accounts for both axial and flexural vibrations and predicts the electro-mechanical (E/M) impedance response, as it would be measured at the piezoelectric active sensor's terminals. Experiments were conducted on simple beam specimens in support of the theoretical investigation. The measured results are well compared with theoretical predictions. Frequency increase with thickness reduction was experimentally confirmed. This observation could be directly used in the detection of corrosion damage in metallic structures, and of disbonding/delaminations in adhesively bonded and composite structures.

The transition of presented results into two-dimensional structures was attempted. Theoretical analysis for circular plates with two sets of boundary conditions was presented. However, exact expression for E/M impedance seen by the sensors has not been derived yet, which gives motivation for further theoretical investigations. The thin plate specimens were designed to study sensor's sensitivity to the presence of structural damage. The experimental results on thin-gauge aluminum plates and real aging aircraft panel were presented to illustrate the method's potential. However, this work is still in process, but preliminary results are very encouraging. Further developments will be directed towards identification of simulated cracks presence and location.

The work reported in this paper has shown that unobtrusive permanently attached PZT active sensors can be successfully used to identify the intrinsic dynamics of a structure through the examination of the recorded E/M impedance spectrum. As presented, the proposed method, using just one active sensor, can only detect structural resonances. The detection of structural mode shapes is also possible, but requires the simultaneous use of several sensors, their number being in direct relationship to the desired modal resolution. The E/M impedance method can be a useful and reliable tool for automatic online structural identification in the ultrasonic frequencies range. Moreover, this method is more convenient to use than traditional modal analysis methods that require separate instrumentation for excitation (e.g., impact hammer) and recording of structural response (e.g., accelerometers). The embedded piezoelectric active sensors are much smaller size, un-obtrusive, and can be permanently attached to the structure, thus permitting in service structural health monitoring.

\section{ACKNOWLEDGMENTS}

The financial support of Department of Energy through the Sandia National Laboratories, contract doc. \# BF 0133 is thankfully acknowledged. Sandia National Laboratories is a multi-program laboratory operated by Sandia Corporation, a Lockheed Martin Company, for the United States Department of Energy under contract DE-AC04-94AL85000.

\section{REFERENCES}

Airey, J., (1911) "The Vibration of Circular Plates and their Relation to Bessel Functions", Proc. Phys. Soc., London, Vol. 23, 1911, pp.225-232.

Ayres, T., Chaudhry Z., and Rogers C., (1996) "Localized Health Monitoring of Civil Infrastructure via Piezoelectric Actuator/Sensor Patches," Proceedings, SPIE's 1996 Symposium on Smart Structures and Integrated systems, SPIE Vol. 2719, pp. 123-131.

Banks, H. T., Smith, R. C., Wang, Y., (1996) “Smart Material Structures: Modeling, Estimation and Control”, Masson, John Wiley \& Sons, Paris 1996.

Blevins, R. D., (1979) “Formulas for Natural Frequency and Mode Shape”, Litton Educational Publishing Inc., 1979.

Broch, J. T. (1984) "Mechanical Vibration and Shock Measurements" Brüel \& Kjær, 1984.

Chaudhry, Z., Joseph, T., Sun, F., and Rogers, C., (1995). "Local-Area Health Monitoring of Aircraft via Piezoelectric Actuator/Sensor Patches," Proceedings, SPIE North American Conference on Smart Structures and Materials, San Diego, CA, 26 Feb. - 3 March, 1995; Vol. 2443, pp. 268-276.

Chaudhry, Z., Sun, F. P, and Rogers C. A., (1994) "Health Monitoring of Space Structures Using Impedance Measurements," Fifth International Conference on Adaptive Structures, Sendai, Japan, 5-7 December, 1994; pp. 584-591.

Clark, R. L., Burdisso, R A., Fuller, C. R., (1993) "Design Approaches for Shaping Polyvinylidene Fluoride Sensors in ACTIVE structural Acoustic Control" Journal of Intelligent Material Systems and Structures, v 4 n 3 Jul 1993 p 354-365, 1993.

Collins, K., Plaut, R., and Wauer, J., (1992) "Free and Forced Longitudinal Vibrations of Cantilevered Bar with a Crack", Journal Vibration Acoustics Stress Reliability Design, 114, pp.171-177, 1992.

Colwell, R.C., (1936) "The Vacuum Tube Oscillator for Membranes and Plates”, Journal of Acoustical Society of America, Vol. 7, 1936, pp.228-230.

Crawley, E. A. and deLuis, J., (1987) "Use of Piezoelectric Actuators as Elements of Intelligent Structures”, AIAA Journal, Vol. 25, No. 10, pp. 1375-1385, 1987.

D'Cruz, J., (1993) “Active Control of Panel Vibrations with Piezoelectric Actuators", Journal of Intelligent Material Systems and Structures, v 4 n 3 Jul 1993 p 398-402, 1993.

Dimitriadis, E. K., Fuller, C. R., Rogers, C. A., (1991) "Piezoelectric Actuators for Distributed Vibration Excitation of Thin Plates", 
Vol.113, Journal of Vibration and Acoustics, 1991.

Ewins, D. J., (1984) "Modal Test: Theory and Practice", Research Studies Press Ltd., Letchworth, Hertfortshire, England, 1984.

Giurgiutiu, V., Reynolds, A., and Rogers, C. A., (1998), "Experimental Investigation of E/M Impedance Health Monitoring of SpotWelded Structural Joints" submitted for publication to the Journal of Intelligent Material Systems and Structures, July 1998.

Giurgiutiu, V., Zagrai, A. (2001b) "Piezoelectric Active Sensors - Theory and Practice", Report \# USC-ME-LAMSS-2001-102, February $26,2001$.

Giurgiutiu, V., Zagrai, A., (2001a), "Embedded Self-Sensing Piezoelectric Active Sensors for On-Line Structural Identification", Submitted to: Transactions of ASME, Journal of Vibration and Acoustics, January 2001.

Giurgiutiu, V.; Redmond, J.; Roach, D.; Rackow, K. (2000a), "Active Sensors for Health Monitoring of Aging Aerospace Structures", Paper \# 3985-32, SPIE's $7^{\text {th }}$ Annual International Symposium on Smart Structures and Materials, 5-9 March 2000, Newport Beach, CA, SPIE Vol. 3985, pp. 294-305.

Giurgiutiu, V.; Rogers, C. A. (2000b) "Modal Expansion Modeling of the Electro-Mechanical (E/M) Impedance Response of 1-D Structures" European COST F3 Conference on System Identification \& Structural Health Monitoring, Universidad Politecnica de Madrid, Spain, 6-9 June 2000

Harris, C., M., (1996) "Shock and Vibration Handbook", McGraw-Hill, USA, 1996.

Heylen, W., Lammens, S., Sas, P., (1997) "Modal Analysis Theory and Testing", Katholieke Universiteit Leuven, Heverrlee, Belgium, 1997.

IEEE Std. 176 (1987), IEEE Standard on Piezoelectricity, The Institute of Electrical and Electronics Engineers, Inc., 1987

Inman, D. J., (1996) "Engineering Vibration", Prentice-Hall, Inc., 1996.

Itao, K., Crandall, S.H. (1979) "Natural Modes and Natural Frequencies of Uniform, Circular, Free-Edge Plates", Journal of Applied Mechanics, Vol. 46, 1979, pp. 448-453.

Kelly, S. G., (2000) "Fundamentals of Mechanical Vibration", $2^{\text {nd }}$ edition , McGraw-Hill, 2000.

Kunukkasseril, V.X., Swamidas, A.S.J. (1974) "Vibration of Continuous Circular Plates", International Journal of Solids Structures, Vol. 10, 1974, pp. 603-619.

Liang, C., Sun, F. P., and Rogers C. A., (1994) "Coupled Electro-Mechanical Analysis of Adaptive Material System-Determination of the Actuator Power Consumption and System energy Transfer”, Journal of Intelligent Material Systems and Structures, Vol. 5, January 1994, pp. 12-20

Liessa, A. (1969) "Vibration of Plates", Published for the Acoustical Society of America through the American Institute of Physics, Reprinted in 1993

Maia, N., Silva, J., He, J., Lieven, N., Lin, R., Skingle, G., To, W., Urgueira, A., (1997) "Theoretical and Experimental Modal Analysis" Research Studies Press Ltd., 1997.

Meirovitch, L., (1986) "Elements of Vibration Analysis", $2^{\text {nd }}$ edition, McGraw-Hill, 1986.

Park, G., Cudney, H. H., Inman, D. J., (2000) “An Integrated Health Monitoring Technique Using Structural Impedance Sensors” Journal of Intelligent Material Systems and Structures, (in press), 2000.

Polytec PI, Inc. (2000), www.polytecpi.com

Rao, J.S. (1999) "Dynamics of Plates". Marcel Dekker, Inc., Narosa Publishing House, 1999

Soedel, W. (1993) "Vibrations of Plates and Shells”, Marcel Dekker, Inc., 1993

Sun, F. P., Liang C., and Rogers, C. A., (1994) "Experimental Modal Testing Using Piezoceramic Patches as Collocated SensorsActuators", Proceeding of the 1994 SEM Spring Conference \& Exhibits, Baltimore, MI, June 6-8, 1994.

Timoshenko, S., P. (1955) "Vibration Problems in Engineering", D.Van Nostrand Company Inc., 1955.

Wah, T., (1962) "Vibration of Circular Plates", Journal of Acoustical Society of America, Vol. 34, 1962, pp.275-281.

Wang, B., Chen, R., (2000) "The Use of Piezoceramic Transducers for Smart Structural Testing”, Proceeding of SPIE 2000 Conference, Newport Beach, CA.

Zhou, S., Liang, C., and Rogers, C., (1996) "An Impedance-Based System Modeling Approach for Induced Strain Actuator-Driven Structures”, Journal of Vibration and Acoustics, July 1996, pp.323-331. 